\title{
Conditionally essential genes for survival during starvation in Enterococcus faecium E745
}

\author{
Vincent de Maat ${ }^{1}$, Sergio Arredondo-Alonso ${ }^{1}$, Rob J. L. Willems ${ }^{1}$ and Willem van Schaik ${ }^{1,2^{*}}$ (D)
}

\begin{abstract}
Background: The nosocomial pathogen Enterococcus faecium can survive for prolonged periods of time on surfaces in the absence of nutrients. This trait is thought to contribute to the ability of E. faecium to spread among patients in hospitals. There is currently a lack of data on the mechanisms that are responsible for the ability of $E$. faecium to survive in the absence of nutrients.
\end{abstract}

Results: We performed a high-throughput transposon mutant library screening (Tn-seq) to identify genes that have a role in long-term survival during incubation in phosphate-buffered saline (PBS) at $20^{\circ} \mathrm{C}$. A total of 24 genes were identified by Tn-seq to contribute to survival in PBS, with functions associated with the general stress response, DNA repair, metabolism, and membrane homeostasis. The gene which was quantitatively most important for survival in PBS was usp (locus tag: EfmE745_02439), which is predicted to encode a $17.4 \mathrm{kDa}$ universal stress protein. After generating a targeted deletion mutant in usp, we were able to confirm that usp significantly contributes to survival in PBS and this defect was restored by in trans complementation. The usp gene is present in $99 \%$ of a set of 1644 E. faecium genomes that collectively span the diversity of the species.

Conclusions: We postulate that usp is a key determinant for the remarkable environmental robustness of $E$. faecium. Further mechanistic studies into usp and other genes identified in this study may shed further light on the mechanisms by which $E$. faecium can survive in the absence of nutrients for prolonged periods of time.

Keywords: Enterococcus faecium, Starvation, Stress response, Functional genomics, Tn-seq

\section{Background}

Enterococcus faecium is a commensal of the human gut, but has emerged over the last few decades as an opportunistic pathogen which causes infections in hospitalized patients. E. faecium infections are often difficult to treat due to the high prevalence of resistance to antibiotics, including virtually all cephalosporins, aminoglycosides, clindamycin, and trimethoprim-sulfamethoxazole [1]. Additionally, resistance to the glycopeptide vancomycin

\footnotetext{
* Correspondence: w.vanschaik@bham.ac.uk

'Department of Medical Microbiology, University Medical Center Utrecht, Heidelberglaan 100, 3584 CX Utrecht, the Netherlands

${ }^{2}$ Institute of Microbiology and Infection, College of Medical and Dental

Sciences, University of Birmingham, Birmingham B15 2TT, UK
}

is increasingly widespread in E. faecium strains, further complicating the treatment of infections. While the accumulation of antibiotic resistance determinants is a major contributor to E. faecium's emergence as an important nosocomial pathogen, other adaptations, like the ability to form biofilms [2-4] and interact with host extracellular matrix and serum components [5], are also widespread in clinical isolates. Genomic analyses have revealed that the majority of recent clinical isolates of $E$. faecium belong to a defined sub-population which was termed clade A1, with strains from other niches, including animals and healthy humans mostly belonging to other clades in the E. faecium population [6-8].

(C) The Author(s). 2020 Open Access This article is licensed under a Creative Commons Attribution 4.0 International License, which permits use, sharing, adaptation, distribution and reproduction in any medium or format, as long as you give appropriate credit to the original author(s) and the source, provide a link to the Creative Commons licence, and indicate if changes were made. The images or other third party material in this article are included in the article's Creative Commons licence, unless indicated otherwise in a credit line to the material. If material is not included in the article's Creative Commons licence and your intended use is not permitted by statutory regulation or exceeds the permitted use, you will need to obtain permission directly from the copyright holder. To view a copy of this licence, visit http://creativecommons.org/licenses/by/4.0/. The Creative Commons Public Domain Dedication waiver (http://creativecommons.org/publicdomain/zero/1.0/) applies to the data made available in this article, unless otherwise stated in a credit line to the data. 
E. faecium also has the ability to persist for long periods of time on synthetic surfaces like table tops, handrails, doorknobs and other medical surfaces [9-11]. The ability of E. faecium to spread via fomites is thus proposed to play a critical role in the inter-patient spread of E. faecium in hospital settings [12-14]. Many nosocomial pathogens are thought to spread via environmental contamination, but E. faecium can survive 3- to 5 -times longer on inanimate objects compared to other Gram-positive nosocomial pathogens, such as Enterococcus faecalis, Staphylococcus aureus, and streptococci [15-17].

In natural environments, bacteria most often exist in a physiological state that is similar to the stationary phase of growth and are adapted to survive harsh conditions $[18,19]$. While survival strategies like sporulation are limited to a subset of bacterial species, most bacteria possess the ability to survive in the complete absence of nutrients (e.g. in water or buffers) for weeks, months and in some cases even years [20,21]. Research into the mechanisms by which bacteria manage to survive these nutrient-limited conditions is currently relatively scarce. Most stress responses upon nutrient deprivation involve the expression or activity of DNA repair systems, transcriptional regulation, metabolic pathways, and the biogenesis of cell walls and membranes [21, 22].

While survival in the absence of nutrients appears to be an important factor in the spread of E. faecium as a nosocomial pathogen, we currently lack an understanding of the genes involved in these processes. For this study, we have elucidated the E. faecium genes involved in survival upon starvation by high-throughput screening of a mariner transposon mutant library ( $\mathrm{Tn}$-seq) of a clinical E. faecium strain upon incubation in a buffer without carbon- or nitrogen-sources at room temperature for seven days. With this study, we generated the first insights into the mechanisms by which E. faecium can survive in the absence of nutrients.

\section{Results}

Effect of nutrients and temperature during prolonged incubation

The vancomycin-resistant, clade $\mathrm{A} 1$ strain $E$. faecium E745 was incubated at either $37^{\circ} \mathrm{C}$ or $20^{\circ} \mathrm{C}$ in the rich medium Brain Heart Infusion broth (BHI) or in phosphate-buffered saline (PBS) for up to 10 days (Fig. 1). By determining viable counts, we showed that cells in BHI were able to survive for a prolonged period of time. Indeed, the stationary phase cultures incubated in BHI at $20^{\circ} \mathrm{C}$, showed no reduction in viable counts during the course of the experiments, while at $37^{\circ} \mathrm{C}$ the cultures were only losing their viability $\left(0.9-\log _{10}\right.$ reduction) after more than 7 days. During incubation in the nutrient-free buffer PBS, significant reductions in viable counts were observed after 2 days of incubation at $37^{\circ} \mathrm{C}$

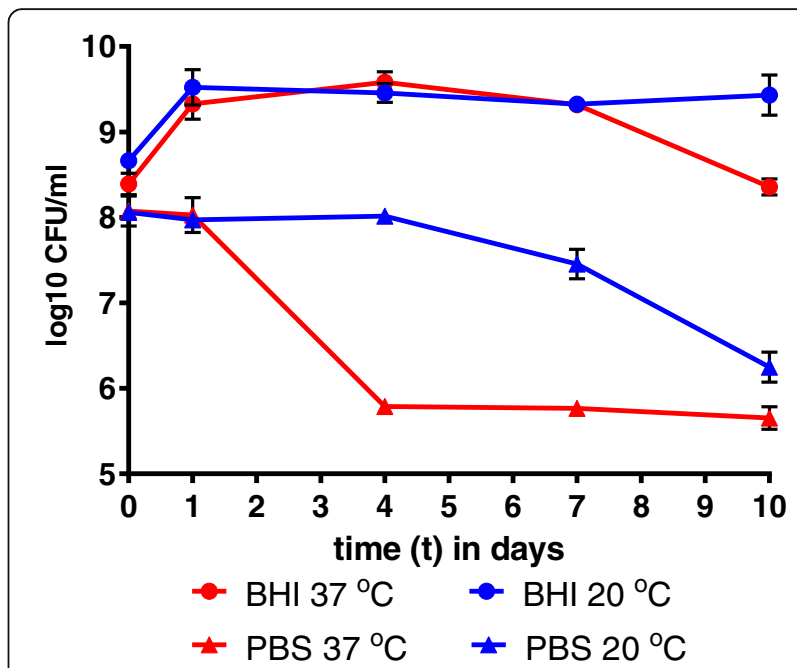

Fig. 1 Survival of E. faecium E745 during starvation. E. faecium strain E745 was incubated for up to 10 days in $\mathrm{BHI}$ (circles) or PBS (triangles) at either $37^{\circ} \mathrm{C}$ (red) or $20^{\circ} \mathrm{C}$ (blue). Error bars show the standard deviation of data from three independent experiments and can be obscured by the symbol

and after 6 days at $20^{\circ} \mathrm{C}$, but a sub-population of cells remained viable until the end of the experiment.

\section{E. faecium E745 genes required for survival during starvation}

To investigate which genes were required for survival in PBS at $20^{\circ} \mathrm{C}$, we incubated a transposon mutant library of E745 in PBS for 7 days at $20^{\circ} \mathrm{C}$. The relative abundance of transposon mutants in 1631 chromosomal and plasmid genes of E. faecium E745 at day 7 was compared to the abundance of mutants present at the start of the experiment (Fig. 2). The abundance of transposon mutants in 24 genes was significantly reduced (BenjaminiHochberg $[\mathrm{BH}]$ corrected $P$-value of $<0.05$ ) by 10 -fold or more, indicating that these genes contribute importantly to the survival of E. faecium in PBS (Table 1). We did not find any mutations that led to a higher abundance after incubation in PBS. The predicted functions of the 24 genes contributing to survival in PBS cover four groups, i.e. general stress response, DNA repair, metabolism, and membrane homeostasis. Transposon mutants in four genes had a greatly reduced (more than a 1000-fold) abundance upon 7-day incubation in PBS, indicating that these genes have a particularly large impact on survival in nutrient-limited conditions.

The usp gene (locus-tag: EfmE745_02439) was identified as the most important gene involved in survival in PBS. Inspection of the mariner transposon insertion sites in usp revealed no unusual patterns in the abundance or placement of the transposon insertion sites, supporting the validity of this hit (Fig. 3a). The usp gene encodes a $17.4 \mathrm{kDa}$ putative universal stress protein with no clear 


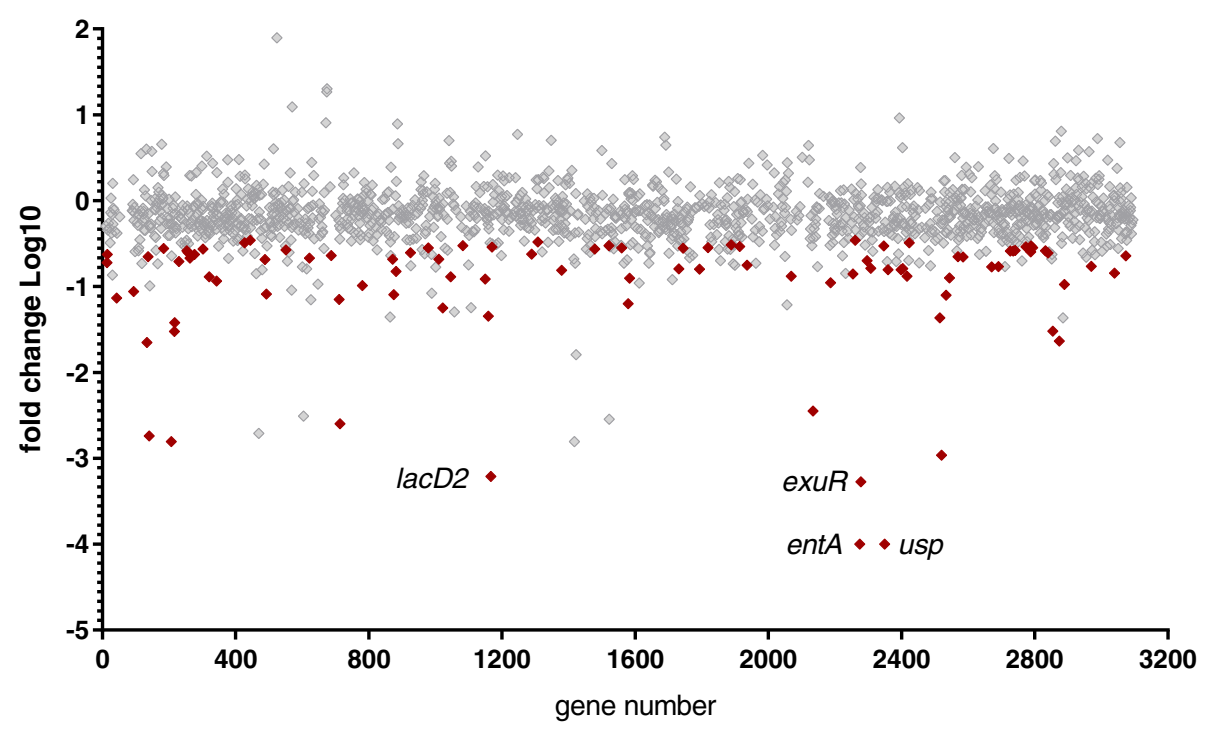

Fig. 2 Tn-seq analysis to identify genes with a role in survival during starvation. Each diamond represents a gene represented in the transposon library. The Y-axis indicates the relative abundance of each gene after incubation in PBS at $20^{\circ} \mathrm{C}$ for 7 days compared to the abundance of each gene at the start of the experiment. A positive value indicates an enrichment of mutants during incubation in PBS while a negative value indicates genes that contribute to survival in PBS, with red diamonds representing genes that passed the threshold for statistical significance. The highlighted dots represent genes that are discussed in the text

function, although homologs are widespread among all domains of life [23, 24]. The second-highest hit in the Tn-seq analyses was found to be in the gene that encodes the previously characterised enterococcal bacteriocin EntA (locus tag: EfmE745_02361) [25, 26]. Like for usp, transposon insertions in entA were no longer detected in the library at day 7. Bacteriocins are small secreted proteins or peptides produced by bacteria to reduce competition of unrelated bacterial strains. A unique feature of bacteriocins is that they are always co-expressed with their associated immunity gene to avoid auto-inhibition [27]. When we inspected the distribution of transposon insertions in ent $A$, we noted that there was only one insertion site in entA which is located close to the $3^{\prime}$ end of the gene (Fig. 3b). As the presumptive bacteriocin immunity gene was found to be overlapping with entA and was also disrupted by the transposon insertion, there is a distinct possibility that this transposon mutant is killed due to the production of the EntA bacteriocin during nutrient deprivation, by the other mutants in the population in which these genes were not disrupted. For this reason, we did not include entA in further functional analyses (described below). The transposon mutants in EfmE745_02364 (exuR) and EfmE745_01205 (lacD2) (Fig. 3c and d) were also highly reduced upon incubation in PBS, and transposon insertion sites in these genes showed no unusual patterns.

Next we studied the presence of all 24 genes identified by $\mathrm{Tn}$-seq in the whole genome sequences of a collection of 1644. E. faecium strains isolated from healthy humans, patients, pets, pigs, and poultry (Fig. 4), previously described in [8]. We found that usp and exuR are present in 99 , and $100 \%$ respectively of the isolates tested and these genes can be thus considered part of $E$. faecium core genome. The lacD2 gene was present in $53 \%$ of genome sequences in this collection. Four genes, including ent $A$, were found to be significantly enriched $\left(X^{2}\right.$ test, $P<0.05$ with Benjamini-Hochberg-correction for multiple testing) in clade A1 strains.

To further test the importance of these genes we have attempted to create gene deletion mutants in usp, exuR and lacD2, but only managed to construct deletions in $u s p$ and exuR. These mutants were tested for their ability to survive in PBS for a 7-day period.

\section{The universal stress protein Usp, but not ExuR, contributes} to survival of $E$. faecium during prolonged starvation

To determine whether usp has an impact on the survival of E745 we created the deletion mutant E745- $\Delta u s p$ and complemented the deletion by supplying the usp gene in trans on a plasmid (strain E745- $\Delta u s p:: u s p-C$ ). We incubated

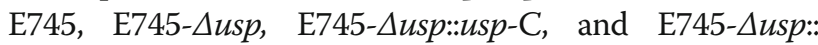
pEF25 (the usp deletion mutant transformed with the empty vector used for in trans complementation) in PBS at $20^{\circ} \mathrm{C}$ and determined viability of the cell suspension over a 7-day period (Fig. 5a). The E. faecium E745 strain showed a similar survival response as seen in Fig. 1. However, the E745-Ausp and E745-Ausp::pEF25 cell suspension lost their viability quicker than $\mathrm{E} 745$, with a statistically significant $(P<0.05$; one way ANOVA) $1-\log$ lower viable count after 7 days than the wild-type. The complemented strain, E745- 
Table 1 Genes with a role in survival during starvation as determined by Tn-seq. Genes of which the transposon mutants were reduced by more than - 10-fold upon incubation in PBS for 7 days, using a BH-adjusted $P$-value of $<0.05$ as cut-off for statistical significance

\begin{tabular}{|c|c|c|c|c|}
\hline Locus Tag & Name & Function & Fold Change & $\begin{array}{l}P \text {-value } \\
\text { (BH adjusted) }\end{array}$ \\
\hline EfmE745_02439 & usp & Putative universal stress protein & $<-2.5 \times 10^{5}$ & 0.023 \\
\hline EfmE745_02361 & entA & Bacteriocin EntA & $<-9.2 \times 10^{4}$ & 0.020 \\
\hline EfmE745_02364 & exuR & DNA-binding transcriptional repressor & $-1.9 \times 10^{3}$ & 0.034 \\
\hline EfmE745_01205 & lacD2 & Tagatose 1,6-diphosphate aldolase 2 & $-1.6 \times 10^{3}$ & 0.018 \\
\hline EfmE745_02646 & sacy & $\begin{array}{l}\text { Levansucrase and sucrase synthesis } \\
\text { operon antiterminator }\end{array}$ & -922.6 & 0.020 \\
\hline EfmE745_00210 & - & hypothetical protein & -639.1 & 0.042 \\
\hline EfmE745_00142 & - & DNA alkylation repair enzyme & -548.8 & 0.034 \\
\hline EfmE745_00731 & licA_1 & $\begin{array}{l}\text { Lichenan-specific phosphotransferase } \\
\text { enzyme IIA component }\end{array}$ & -396.6 & 0.039 \\
\hline EfmE745_02217 & $g b p A$ & GlcNAc-binding protein A precursor & -280.7 & 0.043 \\
\hline EfmE745_00135 & - & Mga helix-turn-helix domain protein & -44.8 & 0.034 \\
\hline EfmE745_03020 & - & hypothetical protein & -43.1 & 0.010 \\
\hline EfmE745_00219 & rhaD & Rhamnulose-1-phosphate aldolase & -33.3 & 0.028 \\
\hline EfmE745_02998 & - & hypothetical protein & -33.1 & 0.010 \\
\hline EfmE745_00220 & rham & L-rhamnose mutarotase & -26.4 & 0.034 \\
\hline EfmE745_02641 & - & $\begin{array}{l}\text { putative HTH-type transcriptional } \\
\text { regulator }\end{array}$ & -23.1 & 0.034 \\
\hline EfmE745_01197 & - & hypothetical protein & -22.1 & 0.023 \\
\hline EfmE745_01055 & - & ABC-2 family transporter protein & -17.8 & 0.040 \\
\hline EfmE745_01627 & - & $\begin{array}{l}\text { PTS system glucitol/sorbitol-specific } \\
\text { transporter subunit IIA }\end{array}$ & -15.9 & 0.016 \\
\hline EfmE745_00728 & $\operatorname{csh} A$ & DEAD-box ATP-dependent RNA helicase & -14.1 & 0.038 \\
\hline EfmE745_00043 & $\operatorname{tmpC}$ & Membrane lipoprotein TmpC precursor & -13.6 & 0.016 \\
\hline EfmE745_02660 & $d r r A$ & $\begin{array}{l}\text { Daunorubicin/doxorubicin resistance } \\
\text { ATP-binding protein }\end{array}$ & -12.6 & 0.046 \\
\hline EfmE745_00895 & $r n m V$ & Ribonuclease M5 & -12.4 & 0.038 \\
\hline EfmE745_00502 & metP & $\begin{array}{l}\text { Methionine import system permease } \\
\text { protein }\end{array}$ & -12.3 & 0.033 \\
\hline EfmE745_00095 & - & $\begin{array}{l}\text { Arsenical resistance operon trans-acting } \\
\text { repressor ArsD }\end{array}$ & -11.4 & 0.029 \\
\hline
\end{tabular}

$\Delta$ usp::usp-C, responded similar to E745 with no statistically significant difference detected at any time point. These results confirm the role of usp in survival of E. faecium E745 in the absence of nutrients. E745- $\Delta$ exuR was found to survive equally well as the wild-type strain during starvation (Fig. 5b), suggesting that exuR does not have a role in survival during starvation.

\section{Discussion}

Most bacteria have a 'feast and famine' lifestyle where long periods of severe nutrient limitation are interrupted by short bursts of nutrient availability [21]. For $E$. faecium, we propose that this cycle is particularly relevant for the spread of this opportunistic pathogen in hospitals, where it can survive on inanimate objects until it is transferred to a human host. Indeed, recent work has suggested that this is a trait that characterizes the genus Enterococcus and has been selected for over hundreds of millions of years of microbial evolution [28]. The finding that the genes most likely to contribute to the survival of E. faecium during starvation are part of the core genome of this species, support the crucial role of starvation tolerance in the evolution of E. faecium. While E. faecium has a low intrinsic virulence, it has become a significant threat to immunocompromised patients in hospitals due to its ability to rapidly acquire genes involved in antibiotic resistance and other traits that contribute to its ability to successfully colonize 

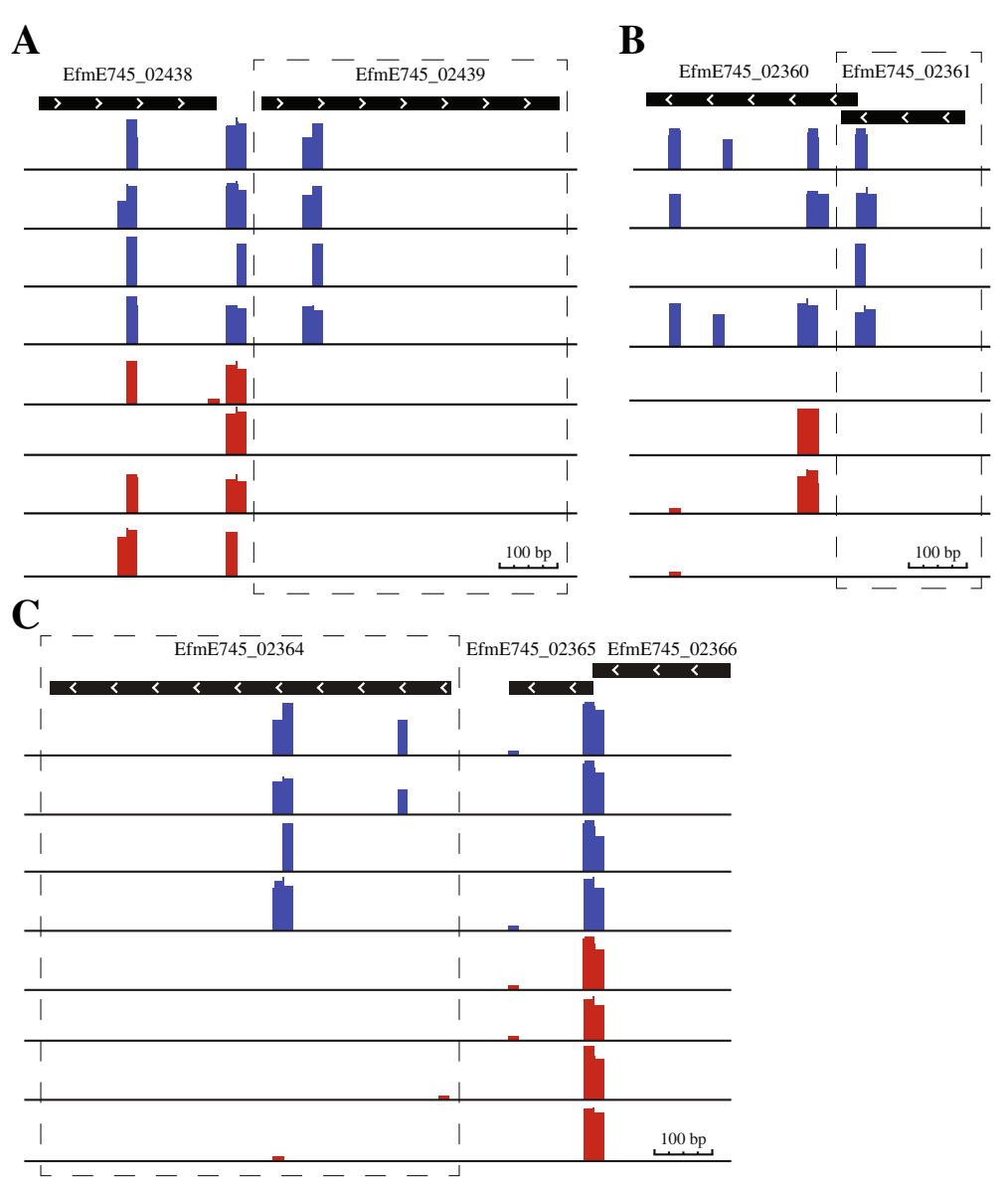

D

EfmE745_01205
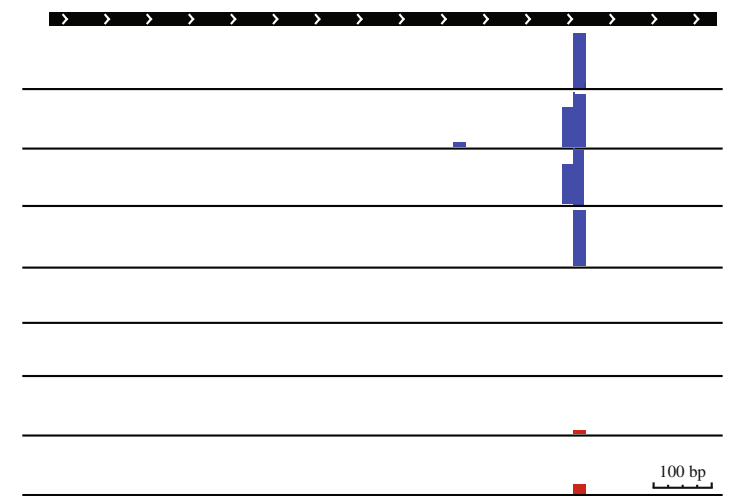

Fig. 3 Visual representation of the transposon insertion sites in usp, entA, exuR and lacD2. Genes and gene direction are depicted by the black bars with arrows. Transposon insertion abundance is shown by the bars below the genes on a $\log _{10}$ scale. Blue and red bars denote the abundance of transposon insertion mutants at day 0 and at day 7, respectively. Abundance of transposon insertion are shown for EfmE745_02439 (usp), EfmE745_02361 (entA), EfmE745_02364 (exuR) and EfmE745_01205 (lacD2) in panel a, b, c and panel d, respectively

patients [29]. The mechanisms by which it can survive and spread in the hospital environment have so far received less attention.

In this study we investigated the potential of vancomycin-resistant Enterococcus faecium to survive in the absence of nutrients. This trait may contribute to the ability of E. faecium to survive on inanimate objects in hospitals and thus can impact its success as a nosocomial pathogen. We noted that in the complete absence of nutrients, during incubation in PBS, E. faecium cultures exhibit a small loss in viability over a period of 7 days at $20^{\circ} \mathrm{C}$, while at $37^{\circ} \mathrm{C}$ a significant loss of 


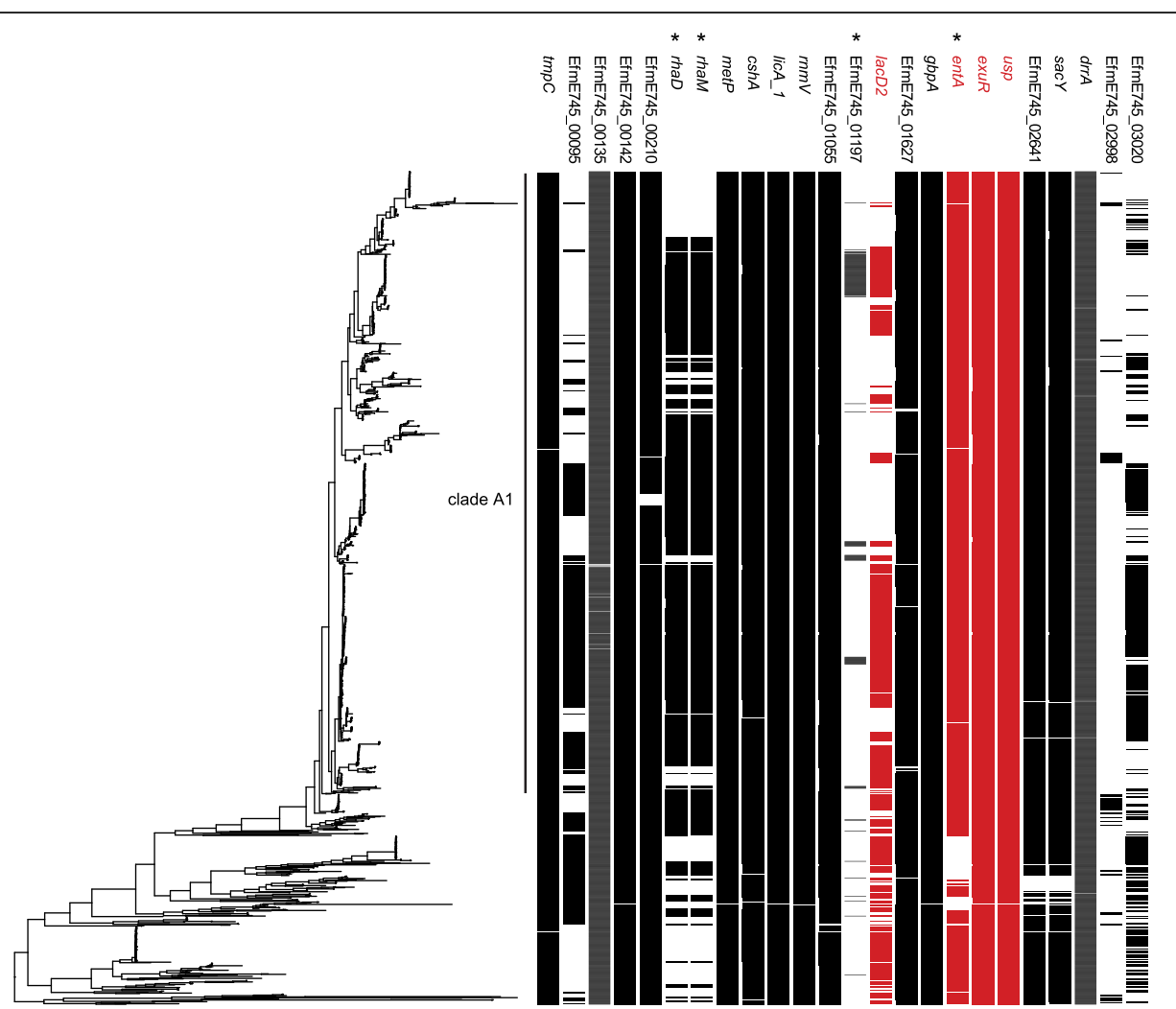

Fig. 4 Presence of genes involved in long-term survival under nutrient-limiting conditions in E. faecium. Whole genome sequence based phylogenetic tree of 1644 E. faecium strains representing the global E. faecium population. The presence of the different E745 genes was plotted along the phylogeny using the R package ggtree. Highlighted in red are EfmE745_01205 (IacD2), EfmE745_02361 (entA), EfmE745_02364 (exuR) and EfmE745_02439 (usp). Genes that are significantly ( $X^{2}$ test, $P<0.05$ with Benjamini-Hochberg-correction for multiple testing) enriched in clade A1 strains versus non-clade $A 1$ strrains are indicated by an asterisk
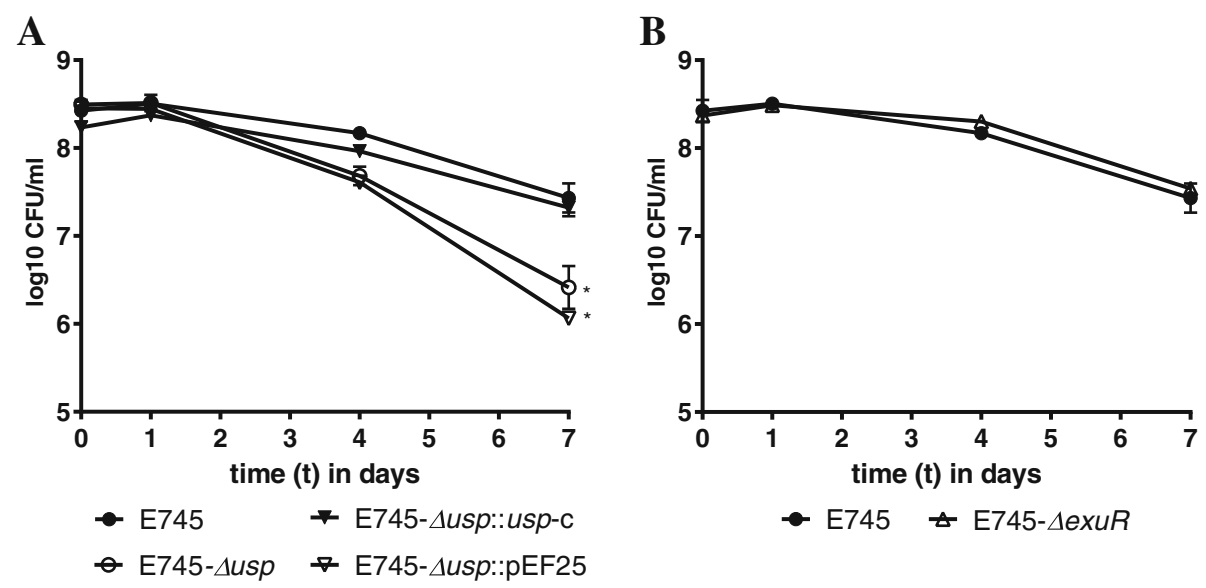

Fig. 5 Phenotypic analysis of usp and exuR deletion mutants. Data for usp and exuR deletion mutants are shown in panel a and $\mathbf{b}$, respectively, and represent three biologically independent experiments. All strains were incubated in PBS at $20^{\circ} \mathrm{C}$ for up to 7 days. ${ }^{*}$ indicates a statistical difference $(P<0.05)$ between survival of E. faecium E745 and $\Delta$ usp and $\Delta$ usp::pEF25 at day 7 , as determined by one way ANOVA. Error bars show the standard deviation of data and can be obscured by the symbol 
viability has occurred after four days, although a subpopulation of cells remains viable, which could represent persister cells [30]. We note that the E. faecium cells may be entering a 'viable but non-culturable' state after prolonged incubation in our experiments [31]. The faster decrease in viability at $37^{\circ} \mathrm{C}$, compared to $20^{\circ} \mathrm{C}$, may be due to increased enzymatic activities and higher metabolic turnover at the higher temperature [32].

To obtain a better understanding into the genes that are involved in the response upon prolonged starvation, we performed $\mathrm{Tn}$-seq on a transposon mutant library that was starved for nutrients for 7 days at $20{ }^{\circ} \mathrm{C}$. This analysis revealed a variety of genes that were potentially involved in retaining viability. Genes could be assigned to four major functional groups, i.e. DNA repair, metabolism, cell membrane and cell wall homeostasis. DNA repair, or lack thereof, can contribute to survival during starvation as reactive oxygen species and other DNA damaging molecules accumulate [33]. Metabolic adaptations during starvation are essential as shifts in metabolism, including changes in transport systems, are required for optimal acquisition of nutrients and the excretion of metabolic waste products [34, 35]. Changes to the cytoplasmic membrane and cell wall have also been described to contribute to the ability of cells to withstand harsh conditions, including starvation [28]. The final category of genes we identified in our Tn-seq analysis have roles in the general stress response, i.e. the cell's extended toolbox for responding to adverse conditions [23, 24]. Our Tn-seq data suggest that most genes involved in survival during nutrient deprivation are part of the core genome of E. faecium. However, four genes are enriched in clade A1 strains which could suggest that these genes may play a role in the emergence of strains from this clade as nosocomial pathogens.

We studied the role of the general stress protein Usp (locus tag: EfmE745_02439) which belongs to this last functional group and confirmed that it has a significant impact on survival of $E$. faecium during starvation. Universal stress proteins (USPs) are mostly studied in $E$. coli where they protect the cell against adverse conditions. However, the exact mechanism(s) by which they act are currently unknown. This could be a result of the many different subtypes found, and the complex responses they contribute to. USPs are found throughout all branches of the tree of life and are important for survival under adverse conditions in many species [23, 24]. In this study we expand these observations to E. faecium.

The exuR gene (locus-tag: EfmE745_02364) appeared to be important for survival upon nutrient starvation on the basis of our Tn-seq screening, but we were unable to confirm this finding by studying an exuR deletion mutant. The E. coli ExuR homologue represses genes involved in the metabolism of D-galacturonate and D-glucuronate
$[36,37]$. ExuR expression is upregulated in the absences of glucose which allows $E$. coli to use different carbon sources for its growth and survival. We speculate that ExuR in E. faecium is involved in rerouting carbohydrate metabolism, similar to $E$. coli. It is possible that the derepression of pathways involved in alternative carbon source utilization in the $\Delta \operatorname{exuR}$ mutant might negatively affect the growth of cells during the recovery step in the glucose-rich medium BHI, which we performed to minimize a Tn-seq signal originating from dead cells in the cell suspension.

\section{Conclusions}

In this study, we have identified 24 E. faecium genes that could potentially affect its survival in the absence of nutrients. Our Tn-seq data suggest that most genes involved in survival during nutrient deprivation are part of the core genome of E. faecium. However, four genes are enriched in clade A1 strains which could suggest that these genes may play a role in the emergence of strains from this clade as nosocomial pathogens. We have confirmed the role of the usp gene, but future functional characterization of other genes identified here may further increase our understanding of the mechanisms by E. faecium can survive outside human or animal hosts.

\section{Methods}

Bacterial strains, plasmids, growth conditions, and oligonucleotides

The vancomycin-resistant, clade A1 E. faecium strain E745 [38] was used throughout this study. E. faecium E745 was isolated from a rectal swab of a patient, during routine surveillance of a VRE outbreak in a Dutch hospital, and its genome was previously sequenced to completion [38]. E. faecium was grown at $37^{\circ} \mathrm{C}$ in brain heart infusion broth (BHI; Oxoid), unless otherwise mentioned. The E. coli strains EC1000 [39] and DH5 $\alpha$ were grown in Lysogeny Broth (LB). Antibiotics were used at the following concentrations: erythromycin $50 \mu \mathrm{g} \mathrm{ml}^{-1}$, chloramphenicol $10 \mu \mathrm{g} \mathrm{ml}^{-1}$, spectinomycin $200 \mu \mathrm{g} \mathrm{ml}^{-1}$ and gentamicin $300 \mu \mathrm{g} \mathrm{ml}^{-1}$ for E. faecium and spectinomycin $100 \mu \mathrm{g} \mathrm{ml}^{-1}$, chloramphenicol $4 \mu \mathrm{g}$ $\mathrm{ml}^{-1}$, erythromycin $100 \mu \mathrm{g} \mathrm{ml}^{-1}$ and gentamicin $50 \mu \mathrm{g}$ $\mathrm{ml}^{-1}$ for E. coli. The transposon library in E. faecium E745 was previously described [38]. The vectors pWS3 [40], pVDM1001 [41], pCRE-Lox [42], pEF25 [43] and pGPA1 [38] were obtained from our laboratory's culture collection. Genomic DNA isolation was performed using the Wizard Genomic DNA Purification kit (Promega).

The sequences of all oligonucleotides used in this study are provided in Table 2 . The sequences of synthesized DNA fragments are listed in Additional file 1. 
Table 2 Oligonucleotides used in this study. Relevant restriction sites are underlined

\begin{tabular}{|c|c|}
\hline Name & Sequence $\left(5^{\prime}-3^{\prime}\right)$ \\
\hline oVDM2001 & AAACACGTTCTTTCCAAACCGTTTCATCCTTTGAG \\
\hline oVDM2002 & AAAACTCAAAGGATGAAACGGTTTGGAAAGAACGT \\
\hline oVDM2003 & CAAACAACGGCATTAACGGAGATTACCAT \\
\hline oVDM2004 & GTGTTTGATAAAAAACTTTGTCTACATGAC \\
\hline oVDM2005 & CTGCATATGCTGTITIAGGCG \\
\hline oVDM2006 & GTGTAATTCTGTCAGAGAGC \\
\hline oVDM2007 & AAAAAAAACCCGGGCAATTGGGGCTATCCATCGGGAAATGAATGCC \\
\hline oVDM2008 & AAAAAAAACTCGAGTTATGACGACGGACTTCTCGC \\
\hline oVDM2009 & GAGGGAATTCTACCGTTCGTATAGCATACATTATACGAAGTTATGATAAACCCAGCGAACCATTTGAGG \\
\hline oVDM2010 & CTCCGAATTCTACCGTTCGTATAATGTATGCTATACGAAGTTATTCAATCTTTATAAGTCCTITTATAA \\
\hline oVDM2011 & GGAGGCAGATTGCCTTGAAT \\
\hline oVDM2012 & TCCAATAATTCGGCTCTCT \\
\hline oVDM2013 & GACTCGTTTATGTTTTGTCCGTC \\
\hline oVDM2014 & GTATITTCCAAGATCGTTCG \\
\hline oVDM2015 & AAAAAAGTCGACACAAGAAAACGGTTCTGTTTCTGCGAGC \\
\hline oVDM2016 & AAAAAAACCCGGGTTAAGCTTTGGTGTGGTCTTTAGCT \\
\hline oVDM2017 & CGGAGCCACGGCGGCCGAA \\
\hline oVDM2018 & CTGCGCGTAATCTGCTGCTTCGA \\
\hline
\end{tabular}

\section{Isolation and transformation of plasmids}

Plasmid isolation from overnight $E$. coli cultures was performed using the GeneJET plasmid miniprep kit (Thermo Fischer Scientific, Bleiswijk, The Netherlands) according to the manufacturer's instructions. Transformation of plasmids into E. faecium E745 was performed as previously described [42].

\section{Determination of viability during starvation}

E. faecium E745 cultures were grown overnight in $3 \mathrm{ml}$ BHI at $37^{\circ} \mathrm{C}$ with shaking at $150 \mathrm{rpm}$. An aliquot of $20 \mu \mathrm{l}$ of an overnight culture was transferred to $20 \mathrm{ml}$ $\mathrm{BHI}$ which was again grown at $37^{\circ} \mathrm{C}$ with shaking at $150 \mathrm{rpm}$ until the culture reached an optical density at $600 \mathrm{~nm}\left(\mathrm{OD}_{600}\right)$ of $0.3-0.4$. Aliquots $(3 \mathrm{ml})$ of these cultures were then centrifuged $(3000 \mathrm{~g}, 5 \mathrm{~min})$, washed once in PBS $(137 \mathrm{mM} \mathrm{NaCl} ; 2.7 \mathrm{mM} \mathrm{KCl} ; 10 \mathrm{mM}$ $\mathrm{Na}_{2} \mathrm{HPO}_{4} ; 2 \mathrm{mM} \mathrm{KH} \mathrm{PO}_{4} ; \mathrm{pH} 7.4$ ) and resuspended in $3 \mathrm{ml}$ PBS and incubated at either $37^{\circ} \mathrm{C}$ or $20^{\circ} \mathrm{C}$ with continuous shaking at $150 \mathrm{rpm}$. Controls remained in $\mathrm{BHI}$ and were incubated at the same temperatures with shaking. At days 0, 1, 4, 7, and 10 samples were taken and serially diluted in PBS, after which viable counts were determined using the Miles and Misra method [44].

Tn-seq analysis of conditionally essential genes in $E$. faecium E745 during prolonged starvation

For the identification of genes that were conditionally essential for prolonged starvation in E. faecium, we grew the previously constructed mariner transposon mutant library in E. faecium E745 [38] in 10-ml BHI supplemented with gentamicin overnight at $37^{\circ} \mathrm{C}$, after which $20 \mu \mathrm{l}$ of the culture was transferred to $10 \mathrm{ml}$ pre-warmed BHI supplemented with $200 \mu \mathrm{g} \mathrm{ml}^{-1}$ gentamicin, which was incubated at $37^{\circ} \mathrm{C}$ until the culture reached an $\mathrm{OD}_{600}$ of 0.3 . After pelleting the cells by centrifugation ( $3000 \mathrm{~g}, 5$ min), washing once with PBS, and resuspension of the cells in $10 \mathrm{ml}$ PBS, a $3 \mathrm{ml}$ aliquot was transferred to a new tube and incubated at $20^{\circ} \mathrm{C}$ for 7 days. The remaining suspension was used for the isolation of genomic DNA. At day 7, the cell suspensions were washed once with PBS and then transferred to $3 \mathrm{ml}$ prewarmed $\mathrm{BHI}$ and incubated at $37^{\circ} \mathrm{C}$ until $\mathrm{OD}_{600} 0.3$ to recover viable cells. The experiments with the transposon mutant library were performed with four independent replicates.

After genomic DNA extraction, Tn-seq libraries were prepared as described previously [38]. Tn-seq libraries were sequenced on one lane of a HiSeq 2500 with $50 \mathrm{nt}$ single-end reads. The sequence reads of the Tn-seq experiments have been made available in the European Nucleotide Archive with accession number PRJEB37076.

\section{Tn-seq data analysis}

Tn-seq data analysis was performed as described previously [38]. In short, Illumina sequence reads were demultiplexed, based on their barcode, using Galaxy [45], and 16-nucleotide fragments of each read, corresponding to E745 genomic sequences flanking the transposon, were 
mapped to the E745 genome using Bowtie 2 [46]. The number of reads per gene were determined using the Integrative Genome Viewer (IGV [47];). Reads that mapped to the final $10 \%$ of a gene were discarded as these insertions may not inactivate gene function [48]. Read counts per gene were then normalized in each replicate by calculating the RPKM (Reads Per Kilobase per Million input reads), with subsequent statistical analysis of these values being performed with Cyber- $T$ $[49,50]$. Genes were determined to be significantly contributing to survival in PBS if the Benjamini-Hochbergcorrected $P$-value was $<0.05$ and the difference in abundance of a gene between day 0 and day 7 was $>10$ or $<-10$.

\section{Construction of targeted deletion mutants}

The usp deletion mutant was created using CRISPRCas9-mediated genome editing as previously described [41]. In short, a CRISPR (Clustered Regularly Interspaced Short Palindromic Repeat) targeting usp was inserted into pVDM1001 by annealing oVDM2001 and oVDM2002 together and ligating this DNA fragment into BsaI-digested pVDM1001 creating pVDM-xusp. Next, a DNA fragment (gBlock, Integrated DNA Technologies; Leuven, Belgium) was synthesized containing the $463 \mathrm{bp}$ upstream region of usp fused together with the $513 \mathrm{bp}$ downstream region of usp (Additional file 1). This DNA fragment was amplified using oVDM2003 and oVDM2004 and was subsequently ligated in SmaI-digested pVDM-xusp creating pVDM- $\Delta u s p$. This plasmid was then transformed into E. faecium E745 which already carried pVPL3004 [51] with selection for transformants on BHI agar containing $200 \mu \mathrm{g} \mathrm{ml}^{-1}$ spectinomycin and $50 \mu \mathrm{g} \mathrm{ml}^{-1}$ erythromycin at $30^{\circ} \mathrm{C}$ for $48 \mathrm{~h}$. The deletion of usp was confirmed by PCR using oVDM2005 and oVDM2006. The plasmids pVPL3004 and pVDM- $\Delta u s p$ were cured from $E$. faecium E745- $\Delta$ usp by sub-culturing in BHI for $72 \mathrm{~h}$.

The 848-bp DNA fragment containing the promotor of usp and the complete usp gene for in trans complementation (Additional file 1) was synthesized, and subsequently this fragment was amplified by PCR using oVDM2015 and oVDM2016, and subsequently digested with XmaI and SalI. The digested fragment was ligated into pEF25 to form pEF25-usp$C$. This vector was subsequently transformed into E745- $\Delta u s p$ and transformants were selected on BHI agar supplemented with $200 \mu \mathrm{g} \mathrm{ml}^{-1}$ spectinomycin. The presence of the vector in E. faecium E745- $\Delta u s p$ was confirmed by PCR using oVDM2017 and oVDM2018 and the resulting complemented strain was named E745- $\Delta$ usp::usp-C.

We were unable to generate an exuR deletion mutant with the CRISPR-based genome editing approach described above and we thus used our previously described [42] allelic replacement method, with minor modifications, to generate this mutant. A DNA fragment (Integrated DNA Technologies; Leuven, Belgium) was ordered containing the $496 \mathrm{bp}$ upstream region of exuR fused to the $515 \mathrm{bp}$ region downstream of exuR (Additional file 1). The DNA fragment was amplified using oVDM2007 and oVDM2008, digested with XhoI and $\mathrm{XmaI}$ and subsequently ligated into similarly digested pWS3 to create pVDM-exuR. The gentamicin resistance cassette from pGPA1 was amplified using oVDM2009 and oVDM2010, followed by digestion with EcoRI. This DNA fragment was then ligated into similarly digested pVDM-exuR, resulting in pVDMexuR + G. E745 was transformed with pVDM-exuR $+G$ and transformants were selected at $30{ }^{\circ} \mathrm{C}$ on $\mathrm{BHI}$ supplemented with $200 \mu \mathrm{g} \mathrm{ml}^{-1}$ spectinomycin. The presence of $\mathrm{pVDM}-\operatorname{exu} R+G$ in the transformants was confirmed by PCR using oVDM2011 and oVDM2012. To induce a double crossover event to replace exuR with the gentamicin resistance cassette, a colony harbouring pVDM-exuR $+G$ was used to inoculate $200 \mathrm{ml}$ BHI medium and then subcultured for $72 \mathrm{~h}$ at $37^{\circ} \mathrm{C}$ after which the culture was plated on BHI with $300 \mu \mathrm{g}$ $\mathrm{ml}^{-1}$ gentamicin. At least 100 colonies were transferred to BHI agar containing either $300 \mu \mathrm{g} \mathrm{ml}^{-1}$ gentamicin or $200 \mu \mathrm{g} \mathrm{ml}^{-1}$ spectinomycin. Colonies that were resistant to gentamicin but not spectinomycin were checked for successful double crossover events using oVDM2013 and oVDM2014. The resulting mutant was named E745:: $\Delta$ exuR $+G$ and was consequently transformed with pCRE-lox to remove the gentamicin cassette from the genome, as described previously [42]. Removal of the gentamicin cassette was confirmed using PCR using oVDM2013 and oVDM2014 and curing of pCRE-lox after removal of the gentamicin cassette was performed as described previously [42].

\section{Presence and absence of genes involved in survival during starvation in $E$. faecium genomes}

Abricate (https://github.com/tseemann/abricate, version 0.8 ) was used to identify presence of the $24 \mathrm{E}$. faecium E745 genes that had the largest role in survival in PBS, as determined by Tn-seq, against the draft assemblies from 1644 E. faecium genomes that represent the global diversity of the species E. faecium [8]. We considered a minimum identity and coverage of 95 and $80 \%$, respectively, to consider a gene as present in a particular draft assembly. To visualize the presence and absence of these genes in the context of the phylogeny of E. faecium, we used the neighbour-joining tree described in [8], based on a RAxML tree-based of 955 E. faecium core genes, and used the $\mathrm{R}$ package ggtree (version 1.14.6) to plot the presence of the different E745 genes. 


\section{Supplementary information}

Supplementary information accompanies this paper at https://doi.org/10. 1186/s12864-020-06984-2.

Additional file 1. Sequences of synthesized DNA fragments for the deletion of usp and exuR and the in trans complementation of usp.

\section{Abbreviations}

PBS: Phosphate-buffered saline; BHI: Brain Heart Infusion broth; BH: Benjamini-Hochberg; USPs: Universal Stress Proteins; LB: Lysogeny Broth; $\mathrm{OD}_{600}$ : Optical density at $600 \mathrm{~nm}$; RPKM: Reads Per Kilobase per Million input reads; IGV: Integrative Genome Viewer; CRISPR: Clustered Regularly Interspaced Short Palindromic Repeat

\section{Acknowledgements}

Not applicable.

\section{Authors' contributions}

VdM performed experiments and analysed the data. SAA contributed bioinformatic analyses. RW and WVS designed the study. VdM and WVS drafted the manuscript. All authors read and approved the final manuscript.

\section{Funding}

This work was supported by a VIDI grant (917.13.357) from the Netherlands Organization for Scientific Research (VIDI: 917.13.357) and a Royal Society Wolfson Research Merit Award (WM160092) to W.v.S. The funders had no role in study design, data collection and analysis, decision to publish, or preparation of the manuscript.

\section{Availability of data and materials}

The datasets generated in the current study are available in the European Nucleotide Archive with accession number PRJEB37076. The genome sequence of E. faecium E745 used in this study is available through the European Nucleotide Archive with accession number GCA_001750885.1. Requests for E. faecium E745 can be made by contacting the corresponding author.

\section{Ethics approval and consent to participate}

Not applicable.

\section{Consent for publication}

Not applicable.

\section{Competing interests}

None to declare.

\section{Received: 16 April 2020 Accepted: 12 August 2020}

Published online: 18 August 2020

\section{References}

1. García-Solache M, Rice LB. The Enterococcus: a model of adaptability to its environment. Clin Microbiol Rev. 2019;32:1-28.

2. Heikens $E$, Singh KV, Jacques-Palaz KD, van Luit-Asbroek M, Oostdijk EAN, Bonten MJM, et al. Contribution of the enterococcal surface protein Esp to pathogenesis of Enterococcus faecium endocarditis. Microbes Infect. 2011;13:1185-90.

3. Heikens E, Bonten MJM, Willems RJL. Enterococcal surface protein esp is important for biofilm formation of Enterococcus faecium E1162. J Bacteriol. 2007;189:8233-40

4. Paganelli FL, Huebner J, Singh KV, Zhang X, van Schaik W, Wobser D, et al. Genome-wide screening identifies Phosphotransferase system permease BepA to be involved in Enterococcus faecium endocarditis and biofilm formation. J Infect Dis. 2016;214:189-95.

5. Zhao M, Sillanpää J, Nallapareddy SR, Murray BE. Adherence to host extracellular matrix and serum components by Enterococcus faecium isolates of diverse origin. FEMS Microbiol Lett. 2009:301:77-83.

6. Lebreton F, van Schaik W, McGuire AM, Godfrey P, Griggs A, Mazumdar V, et al. Emergence of epidemic multidrug-resistant Enterococcus faecium from animal and commensal strains. MBio. 2013;4:00534-13.
7. Raven KE, Reuter S, Reynolds R, Brodrick HJ, Russell JE, Török ME, et al. A decade of genomic history for healthcare-associated Enterococcus faecium in the United Kingdom and Ireland. Genome Res. 2016;26:1388-96.

8. Arredondo-Alonso S, Top J, Schürch AC, McNally A, Puranen S, Pesonen M, et al. Plasmids shaped the recent emergence of the major nosocomial pathogen Enterococcus faecium. MBio. 2020;11:530725.

9. Wagenvoort JHT, De Brauwer EIGB, Penders RJR, van der Linden CJ, Willems RJ, Top J, et al. Environmental survival of vancomycin-sensitive ampicillinresistant Enterococcus faecium (AREfm). Eur J Clin Microbiol Infect Dis. 2015; 34:1901-3.

10. Wagenvoort JHT, De Brauwer EIGB, Penders RJR, Willems RJ, Top J, Bonten MJ. Environmental survival of vancomycin-resistant Enterococcus faecium. Hosp Infect. 2011;77:282-3.

11. Weinstein RA, Hota B. Contamination, disinfection, and cross-colonization: are hospital surfaces reservoirs for nosocomial infection? Clin Infect Dis. 2004;39:1182-9.

12. Farr BM. Prevention and control of methicillin-resistant Staphylococcus aureus infections. Curr Opin Infect Dis. 2004;17:317-22.

13. O'Connell $\mathrm{NH}$, Humphreys $\mathrm{H}$. Intensive care unit design and environmental factors in the acquisition of infection. J Hosp Infect. 2000;45:255-62.

14. Gao W, Howden BP, Stinear TP. Evolution of virulence in Enterococcus faecium, a hospital-adapted opportunistic pathogen. Curr Opin Microbiol. 2018;41:76-82.

15. de Regt MJA, van der Wagen LE, Top J, Blok HEM, Hopmans TEM, Dekker AW, et al. High acquisition and environmental contamination rates of CC17 ampicillin-resistant Enterococcus faecium in a Dutch hospital. J Antimicrob Chemother. 2008;62:1401-6.

16. Wagenvoort JH, Penders RJ. Long-term in-vitro survival of an epidemic MRSA phage-group III-29 strain. J Hosp Infect. 1997;35:322-5.

17. Wagenvoort JHT, Joosten EJAJ. An outbreak Acinetobacter baumannii that mimics MRSA in its environmental longevity. J Hosp Infect. 2002;52:226-7.

18. Finkel SE. Long-term survival during stationary phase: evolution and the GASP phenotype. Nat Rev Microbiol. 2006;4:113-20.

19. Leclercq R, Oberlé K, Galopin S, Cattoir V, Budzinski H, Petit F. Changes in enterococcal populations and related antibiotic resistance along a medical center-wastewater treatment plant-river continuum. Appl Environ Microbiol. 2013;79:2428-34.

20. Liao $\mathrm{CH}$, Shollenberger LM. Survivability and long-term preservation of bacteria in water and in phosphate-buffered saline. Lett Appl Microbiol. 2003;37:45-50.

21. Navarro Llorens JM, Tormo A, Martínez-García E. Stationary phase in gramnegative bacteria. FEMS Microbiol Rev. 2010;34:476-95.

22. Boutte CC, Crosson S. Bacterial lifestyle shapes stringent response activation. Trends Microbiol. 2013;21:174-80

23. Kvint K, Nachin L, Diez A, Nyström T. The bacterial universal stress protein: function and regulation. Curr Opin Microbiol. 2003;6:140-5.

24. Seifart Gomes C, Izar B, Pazan F, Mohamed W, Mraheil MA, Mukherjee K, et al. Universal stress proteins are important for oxidative and acid stress resistance and growth of Listeria monocytogenes EGD-e in vitro and in vivo. PLoS One. 2011;6:e24965.

25. O'Keeffe T, Hill C, Ross RP. Characterization and heterologous expression of the genes encoding enterocin a production, immunity, and regulation in Enterococcus faecium DPC1146. Appl Environ Microbiol. 1999:65:1506-15.

26. Henning C, Gautam D, Muriana P. Identification of multiple bacteriocins in Enterococcus spp. using an Enterococcus-specific bacteriocin PCR array. Microorganisms. 2015;3:1-16.

27. Hickey RM, Twomey DP, Ross RP, Hill C. Potential of the enterocin regulatory system to control expression of heterologous genes in Enterococcus. J Appl Microbiol. 2003;95:390-7.

28. Lebreton F, Manson AL, Saavedra JT, Straub TJ, Earl AM, Gilmore MS. Tracing the enterococci from paleozoic origins to the hospital. Cell. 2017:169:849-61 e13.

29. Guzman Prieto AM, van Schaik W, Rogers MRC, Coque TM, Baquero F, Corander J, et al. Global emergence and dissemination of enterococci as nosocomial pathogens: attack of the clones? Frontiers Microbiol. 2016;7:788.

30. Van den Bergh B, Fauvart M, Michiels J. Formation, physiology, ecology, evolution and clinical importance of bacterial persisters. FEMS Microbiol Rev. 2017:41:219-51.

31. Del Mar LM, Bonato B, Signoretto C, Canepari P. Vancomycin resistance is maintained in enterococci in the viable but nonculturable state and after division is resumed. Antimicrob Agents Chemother. 2003;47:1154-6. 
32. Arana I, Muela A, Orruño M, Seco C, Garaizabal I, Barcina I. Effect of temperature and starvation upon survival strategies of Pseudomonas fluorescens CHAO: comparison with Escherichia coli. FEMS Microbiol Ecol. 2010;74:500-9.

33. Saint-Ruf C, Pesut J, Sopta M, Matic I. Causes and consequences of DNA repair activity modulation during stationary phase in Escherichia coli. Crit Rev Biochem Mol Biol. 2007;42:259-70.

34. Crosse AM, Greenway DLA, England RR. Accumulation of ppGpp and ppGp in Staphylococcus aureus 8325-4 following nutrient starvation. Lett Appl Microbiol. 2000;31:332-7.

35. Liebeke M, Dörries K, Zühlke D, Bernhardt J, Fuchs S, Pané-Farré J, et al. A metabolomics and proteomics study of the adaptation of Staphylococcus aureus to glucose starvation. Mol BioSyst. 2011;7:1241-53.

36. Robert-Baudouy J, Portalier R, Stoeber F. Regulation of hexuronate system genes in Escherichica coli K-12: multiple regulation of the uxu operon by exuR and uxuR gene products. J Bacteriol. 1981;145:211-20.

37. Hugovieux-Cotte-Pattat N, Robert-Baudouy J. Regulation and transcription direction of exuR, a self-regulated repressor in Escherichia coli K-12. J Mol Biol. 1982;156:221-8.

38. Zhang $X$, de Maat V, Guzmán Prieto AM, Prajsnar TK, Bayjanov JR, de Been $M$, et al. RNA-seq and Tn-seq reveal fitness determinants of vancomycinresistant Enterococcus faecium during growth in human serum. BMC Genomics. 2017;18:893.

39. Leenhouts K, Buist G, Bolhuis A, ten Berge A, Kiel J, Mierau I, et al. A general system for generating unlabelled gene replacements in bacterial chromosomes. Mol Gen Genet. 1996;253:217-24.

40. Zhang X, Vrijenhoek JEP, Bonten MJM, Willems RJL, van Schaik W. A genetic element present on megaplasmids allows Enterococcus faecium to use raffinose as carbon source. Environ Microbiol. 2011;13:518-28.

41. de Maat V, Stege PB, Dedden M, Hamer M, van Pijkeren J-P, Willems RJL, et al. CRISPR-Cas9-mediated genome editing in vancomycin-resistant Enterococcus faecium. FEMS Microbiol Lett. 2019;366:fnz256.

42. Zhang X, Paganelli FL, Bierschenk D, Kuipers A, Bonten MJM, Willems RJL, et al. Genome-wide identification of ampicillin resistance determinants in Enterococcus faecium. PLoS Genet. 2012;8:e1002804.

43. Top J, Sinnige JC, Majoor EAM, Bonten MJM, Willems RJL, Van Schaik W. The recombinase IntA is required for excision of esp-containing ICEEfm1 in Enterococcus faecium. J Bacteriol. 2011;193:1003-6.

44. Miles AA, Misra SS, Irwin JO. The estimation of the bactericidal power of the blood. J Hyg (Lond). 1938;38:732-49.

45. Afgan E, Baker D, Batut B, Van Den Beek M, Bouvier D, Ech M, et al. The galaxy platform for accessible, reproducible and collaborative biomedical analyses: 2018 update. Nucleic Acids Res. 2018;46:W537-44.

46. Langmead B, Trapnell C, Pop M, Salzberg SL. Ultrafast and memory-efficient alignment of short DNA sequences to the human genome. Genome Biol. 2009;10:R25.

47. Robinson JT, Thorvaldsdóttir H, Winckler W, Guttman M, Lander ES, Getz G, et al. Integrative genomics viewer. Nat Biotechnol. 2011;29:24-6.

48. Chao MC, Abel S, Davis BM, Waldor MK. The design and analysis of transposon insertion sequencing experiments. Nat Rev Microbiol. 2016; 14:119-28.

49. Baldi P, Long AD. A Bayesian framework for the analysis of microarray expression data: regularized t-test and statistical inferences of gene changes. Bioinformatics. 2001;17:509-19.

50. Kayala MA, Baldi P. Cyber-T web server: differential analysis of highthroughput data. Nucleic Acids Res. 2012;40 Web Server issue:W553-9.

51. Oh JH, Van Pijkeren JP. CRISPR-Cas9-assisted recombineering in Lactobacillus reuteri. Nucleic Acids Res. 2014;42:1-11.

\section{Publisher's Note}

Springer Nature remains neutral with regard to jurisdictional claims in published maps and institutional affiliations.

Ready to submit your research? Choose BMC and benefit from:

- fast, convenient online submission

- thorough peer review by experienced researchers in your field

- rapid publication on acceptance

- support for research data, including large and complex data types

- gold Open Access which fosters wider collaboration and increased citations

- maximum visibility for your research: over $100 \mathrm{M}$ website views per year

At BMC, research is always in progress.

Learn more biomedcentral.com/submissions 